\title{
Valor Prognóstico de Níveis Elevados de Troponina I Isolados em Pacientes sem Síndrome Coronariana Aguda Admitidos no Serviço de Emergência
}

\author{
Prognostic Value of Isolated Elevated Troponin I Levels in Patients without Acute Coronary Syndrome \\ Admitted to the Emergency Department
}

Célia Domingues, ${ }^{1 \oplus}$ Maria João Vidigal Ferreira, ${ }^{1,2}$ Joana Moura Ferreira, ${ }^{1}$ Ana Vera Marinho, ${ }^{\circledR}$ Patrícia Marques Alves, ${ }^{1 \oplus}$ Cátia Ferreira, ${ }^{1 \oplus}$ Isabel Fonseca, ${ }^{1}$ Lino Gonçalves ${ }^{1,2}$

Centro Hospitalar e Universitário de Coimbra EPE, ${ }^{1}$ Coimbra - Portugal

Universidade de Coimbra - Faculdade de Medicina, ${ }^{2}$ Coimbra - Portugal

\section{Resumo}

Fundamento: Embora a elevação não isquêmica da troponina seja frequentemente observada em pacientes admitidos no pronto-socorro (PS), não há consenso quanto ao seu manejo.

Objetivos: Este estudo teve como objetivo caracterizar os pacientes admitidos no PS com elevação da troponina nãoisquêmica e identificar potenciais preditores de mortalidade nessa população.

Métodos: Este estudo observacional retrospectivo incluiu pacientes do PS com resultado positivo no teste da troponina entre junho e julho de 2015. Pacientes com diagnóstico clínico de síndrome coronariana aguda (SCA) foram excluídos. Os dados demográficos dos pacientes e as variáveis clínicas e laboratoriais foram extraídos dos prontuários médicos. Os dados do seguimento foram obtidos por 16 meses ou até a ocorrência de morte. O nível de significância estatística foi de 5\%.

Resultados: A elevação da troponina sem SCA foi encontrada em 153 pacientes no PS. A mediana (IIQ) de idade dos pacientes foi de 78 (19) anos, $80(52,3 \%)$ eram do sexo feminino e $59(38,6 \%)$ morreram durante o seguimento. A mediana do período de seguimento (IIQ) foi de 477 (316) dias. Os sobreviventes eram significativamente mais jovens 76 (24) vs. 84 (13) anos; $p=0,004$ ) e apresentaram uma maior proporção de elevação da troponina isolada (sem elevação da creatina quinase ou mioglobina) em duas avaliações consecutivas: 48 (53,9\%) vs. 8 (17,4\%), p<0,001. Os sobreviventes também apresentaram menor taxa de tratamento antiplaquetário e internação no mesmo dia. Na regressão logística multivariada com ajuste para variáveis significativas na análise univariada, a elevação isolada da troponina em duas avaliações consecutivas mostrou hazard ratio = 0,43 (IC95\% 0,17-0,96, p=0,039); hospitalização, tratamento antiplaquetário anterior e idade permaneceram independentemente associados à mortalidade.

Conclusões: A elevação isolada da troponina em duas medidas consecutivas foi um forte preditor de sobrevida em pacientes no PS com elevação da troponina, mas sem SCA. (Arq Bras Cardiol. 2021; 116(5):928-937)

Palavras-chave: Troponina I; Prognóstico; Pronto-socorro; Lesão Miocárdica Não Isquêmica.

\begin{abstract}
Background: Although non-ischemic troponin elevation is frequently seen in patients admitted to the emergency department (ED), consensus regarding its management is lacking.

Objectives: This study aimed to characterize patients admitted to the ED with non-ischemic troponin elevation and to identify potential mortality predictors in this population.

Methods: This retrospective observational study included ED patients with a positive troponin test result between June and July of 2015. Patients with a clinical diagnosis of acute coronary syndrome (ACS) were excluded. Data on patient demographics and clinical and laboratory variables were extracted from medical records. Follow-up data were obtained for 16 months or until death occurred. The statistical significance level was 5\%.
\end{abstract}

Results: Troponin elevation without ACS was found in 153 ED patients. The median (IQR) patient age was 78 (19) years, 80 (52.3\%) were female and 59(38.6\%) died during follow-up. The median (IQR) follow-up period was 477(316) days. Survivors were significantly younger 76 (24) vs. 84 (13) years; $p=0.004$ ) and featured a higher proportion of isolated troponin elevation (without creatine kinase or myoglobin elevation) in

Correspondência: Célia Domingues •

Centro Hospitalar e Universitário de Coimbra EPE - Praceta Prof Mota Pinto Coimbra 3000-075 - Portugal

E-mail: celiadomingues@me.com

Artigo recebido em 06/06/2019, revisado em 10/03/2020, aceito em 08/04/2020

DOI: DOI: https://doi.org/10.36660/abc.20190356 
two consecutive evaluations: 48 (53.9\%) vs. 8 (17.4\%), p<0.001. Survivors also presented a lower rate of antiplatelet treatment and sameday hospitalization. In the multivariate logistic regression with adjustment for significant variables in the univariate analysis, isolated troponin elevation in two consecutive evaluations showed a hazard ratio $=0.43(95 \% \mathrm{Cl} 0.17-0.96, p=0.039)$; hospitalization, previous antiplatelet treatment and age remained independently associated with mortality.

Conclusions: Isolated troponin elevation in two consecutive measurements was a strong predictor of survival in ED patients with troponin elevation but without ACS. (Arq Bras Cardiol. 2021; 116(5):928-937)

Keywords: Troponin I; Prognosis; Emergency Department; Myocardial Non-Ischemic Injury.

Full texts in English - http://www.arquivosonline.com.br

\section{Introdução}

O infarto do miocárdio (IM) clínico, de acordo com a quarta definição universal, requer a presença de lesão miocárdica aguda detectada por biomarcadores cardíacos anormais associados a evidências de isquemia miocárdica aguda. A troponina cardíaca (Tnc) acima do ponto de corte do percentil 99, com padrão crescente ou decrescente, é o biomarcador de lesão miocárdica, ${ }^{1}$ porque não pode ser liberada por tecidos não cardíacos e tem excelente acurácia para o diagnóstico de infarto agudo do miocárdio. ${ }^{1-3}$

A Tnc é uma proteína distribuída no citoplasma e sarcômero de um miócito cardíaco, principalmente no retículo sarcoplasmático. Três subunidades constituem o complexo da troponina, um componente inibitório (troponina I), componente de ligação à tropomiosina (troponina T) e componente de ligação de cálcio (troponina C). ${ }^{4}$

As subunidades T e I (TncT e Tncl, respectivamente) são específicas do músculo cardíaco e, portanto, podem atuar como marcadores adequados de lesão cardíaca. A TncT apresenta uma descarga dupla, primeiro o componente citoplasmático e depois o componente de ligação. ${ }^{5} \mathrm{~A}$ Tncl é específica para o coração, e não foi identificada no músculo esquelético. Esta especificidade de 100\% mostra que a Tncl pode ser um marcador de necrose miocárdica (MNM) ideal. ${ }^{6}$

Antes do advento da troponina, os MNM anteriores utilizados eram a creatina quinase-isoenzima muscular/ cerebral (CK-MB) e a mioglobina, que eram menos sensíveis e não específicas para IM. ${ }^{7,8}$ Por essa falta de sensibilidade e especificidade, elas foram progressivamente excluídas das investigações de CSA. ${ }^{2,3}$

Embora as subunidades Tnc sejam fortemente específicas para miócitos cardíacos, elas podem ser liberadas sob um amplo espectro de condições patológicas não cardíacas, como sepse, doença renal crônica, emergências hipertensivas, sangramento gastrointestinal, acidente vascular cerebral e rabdomiólise. ${ }^{6,9}$ Nesse cenário, a detecção da troponina pode ser o resultado de 5-8\% da liberação do componente citosólico em resposta ao turnover celular do miócito, liberação celular de produtos de degradação e aumento da permeabilidade da parede celular. ${ }^{10}$

O uso generalizado de ensaios de troponina no prontosocorro (PS) pode representar um desafio diagnóstico difícil quando o teste é anormal em paciente sem SCA. ${ }^{11}$ De acordo com a literatura, níveis elevados de Tnc em pacientes sem SCA foram associados a um prognóstico ruim. ${ }^{10,12-16}$

\section{Objetivo}

Este estudo teve como objetivo identificar os fatores preditivos de mortalidade/sobrevida em pacientes de PS sem SCA e Tncl elevada utilizando as características do paciente, histórico clínico, comorbidades e valores analíticos (incluindo creatinina, CK-MB, mioglobina e Tncl) medidos no PS.

\section{Métodos}

\section{Desenho, local e participantes do estudo}

Neste estudo retrospectivo, analisamos os dados laboratoriais de pacientes consecutivos que vieram ao PS de um hospital universitário da comunidade durante o período de 1 mês, de junho a julho de 2015, e selecionamos pacientes que apresentaram elevação da Tncl.

Todas as informações clínicas foram coletadas, incluindo registros médicos e de enfermagem do PS e hospitalizações, análises e outros exames complementares. O seguimento foi realizado através de registros locais e nacionais para observar as taxas de reinternação cardiovascular e morte.

Os dados coletados incluíram dados demográficos, fatores de risco cardiovascular, ensaio de biomarcador miocárdico e resultados da creatinina, sintoma principal no PS, diagnóstico final do PS, hospitalização, mortalidade da hospitalização, taxa de mortalidade em 30 dias e 16 meses e taxa de reinternação cardiovascular.

Neste estudo, os critérios para o diagnóstico de infarto agudo do miocárdio foram: aumento ou diminuição da Troponina I com pelo menos um valor anormal acima do limite superior de referência do ensaio e, ao menos um dos seguintes: 1) sintomas de isquemia; 2) novas alterações da onda T / segmento ST ou novo bloqueio de ramo esquerdo; 3) desenvolvimento de ondas Q patológicas na eletrocardiografia; 4) nova perda de miocárdio viável ou anormalidades regionais de movimento da parede na imagem; ou 5) identificação de um trombo intracoronário na imagem. ${ }^{1}$

Os registros do PS e a hospitalização de todos os pacientes com Tncl elevada foram revisados pelos investigadores, e os pacientes foram divididos em dois grupos: aqueles com diagnóstico de SCA (IM tipo 1 ou tipo 2 com sinais e sintomas isquêmicos (com vasoespasmo, embolia e dissecção coronária não-aterosclerótica) (grupo A) e aqueles sem SCA com um teste de troponina positivo devido ao desequilíbrio de oferta/ demanda de oxigênio ou lesão miocárdica sem sinais ou sintomas de isquemia miocárdica aguda (grupo B). Pacientes com IM tipo 4 ou 5 não foram incluídos neste estudo, pois, 
por definição, estes não são os pacientes regulares do PS e os pacientes com IM tipo 3 não têm medida de Tncl. ${ }^{1}$

Pacientes sem SCA foram identificados através de critérios predefinidos que incluíram o seguinte: 1) miocardite/ cardiomiopatia: diagnóstico à alta hospitalar ou achados sugestivos de miocardite em teste de imagem ou patológico, cardiomiopatias infiltrativas, como amiloidose ou sarcoidose, uma fração de ejeção $\leq 30 \%$ antes da admissão, ou transplante cardíaco anterior; 2) infecções: condições com impacto sistêmico, como celulite, pneumonia, sepse e pielonefrite; 3) disritmias agudas não relacionadas com a SCA; 4) doença renal crônica ou aguda: doença renal crônica estágio 5, diálise crônica, receptor de transplante renal ou doença renal aguda moderada a grave; 5) patologia do sistema nervoso central: acidente vascular cerebral, convulsão ou hemorragia subaracnóidea; 6) sangramento abdominal ou gastrointestinal agudo; 7) embolia pulmonar; 8) síncope inexplicada; 9) asma ou exacerbação de doença pulmonar obstrutiva crônica; e 10) outros: nível elevado de troponina de etiologia desconhecida que não atende a nenhum dos critérios acima mencionados.

O endpoint primário para este estudo foi a mortalidade durante a hospitalização, em 30 dias e 16 meses, enquanto o endpoint secundário foi a reinternação por doença cardiovascular durante o seguimento.

O seguimento foi concluído em 16 meses ou em caso de morte. O seguimento foi realizado através de consulta aos prontuários eletrônicos e ao registro nacional de óbitos online. O conselho de revisão institucional aprovou o protocolo do estudo. A exigência de consentimento informado foi dispensada porque os pacientes não receberam qualquer tipo de cuidado diferente por causa do estudo.

\section{Ensaios de marcadores de necrose miocárdica}

O nível de troponina I foi determinado utilizando o mesmo imunoensaio Tncl padrão (Troponina I Siemens Dimension EXL) $)^{17-19}$ em todos os pacientes. O teste foi realizado no laboratório central do hospital. Os limites de detecção inferior e superior estabelecidos pelo fabricante foram $0,017 \mathrm{ng} / \mathrm{mL}$ e $4000 \mathrm{ng} / \mathrm{mL}$, respectivamente. As medidas abaixo do limite de detecção receberam o valor de 0 . Os resultados do teste de troponina I foram considerados positivos se o nível fosse superior ao limite de referência $(>0,059 \mathrm{ng} / \mathrm{mL})$ usado no laboratório do PS. Os resultados dos ensaios de CK-MB e mioglobina foram considerados normais quando $<3,6 \mathrm{ng} /$ $\mathrm{mL}$ e 9-82 ng/mL, respectivamente.

Medidas repetidas dos MNM foram realizadas pelo menos 3 horas após a primeira avaliação.

Utilizando a primeira e a segunda avaliação da Tnc, a variação foi calculada da seguinte forma: variação da troponina $\%=(($ segunda troponina $\times 100) /$ primeira troponina $)$ $\times 100 \%$.

\section{Métodos estatísticos}

Todos os dados contínuos foram testados quanto à normalidade com o teste de Shapiro-Wilks; todos apresentaram distribuição não-normal e são apresentados por sua mediana e intervalo interquartil. O teste de Mann-
Whitney foi aplicado para comparar variáveis contínuas. As variáveis categóricas foram representadas por sua frequência e comparadas utilizando o teste exato de Fisher ou o teste de qui-quadrado.

A sobrevida foi analisada utilizando modelos de risco proporcional de Cox uni- e multivariados. Os resultados foram expressos como hazard ratio (HR) com intervalos de confiança de 95\% (IC 95\%). Para as variáveis independentes preditoras de sobrevida, um gráfico de sobrevida foi obtido utilizando o método de Kaplan-Meier e o teste de log-rank. O nível de significância estatística foi estabelecido com um valor de $\mathrm{p}<0,05$. Todas as análises estatísticas foram realizadas utilizando o software SPSS 23.0 para Mac (SPSS, Inc; Chicago, IL, EUA).

\section{Resultados}

\section{Características basais e diagnósticos da população de estudo}

Durante o período de estudo de 1 mês, 10.564 pacientes foram admitidos no PS da nossa instituição. Os pacientes que foram submetidos a avaliações de MNM, incluindo troponina I, CK-MB e mioglobina e sua distribuição de acordo com o status da troponina e o diagnóstico final, estão descritos na Figura 1. Os pacientes foram divididos em dois grupos: Grupo A ( $n=42$ [21,5\%]) com SCA (todos com oclusão/suboclusão aguda de artérias coronárias: 4 deles com IM tipo 2 ( 2 casos de dissecção coronária e 2 de trombose coronária embólica); de 38 com IM tipo 1, 14 apresentavam IAM com supradesnivelamento do segmento ST (IAMCSST); enquanto no Grupo B ( $\mathrm{n}=153$ [78,5\%]) sem SCA, 58 apresentavam desequilíbrio entre oferta/demanda de oxigênio, 53 tinham lesão miocárdica aguda sem sinais ou sintomas de isquemia e 42 tinham elevação estável da Tncl (variação da Tncl em duas análises consecutivas $\leq 20 \%$ ). Entre os pacientes sem SCA, a primeira medida de MNM foi realizada após uma mediana de 6 (IIQ 4) horas desde o início dos sintomas, e 90 pacientes repetiram a medida de MNM após uma mediana de 5 (IIQ 3) horas a partir da primeira avaliação.

Na primeira avaliação do MNM do grupo B, 81 pacientes apresentaram elevação de CK-MB e/ou mioglobina (16 apresentaram elevação de CK-MB, e 40 de mioglobina, enquanto 25 tiveram elevação de ambos). Na segunda avaliação, 18 apresentaram elevação de ambos e 6 apresentaram elevação isolada do CK-MB e 31 da mioglobina. Em ambas as avaliações, 88 pacientes apresentaram pelo menos uma elevação de CKMB e / ou mioglobina.

Os pacientes com resultado positivo no teste da troponina apresentaram um amplo espectro de sintomas clínicos na apresentação (Figura 2). Como esperado, os pacientes que ao final receberam o diagnóstico de SCA (Grupo A) apresentaram maior proporção de dor torácica como queixa principal na apresentação hospitalar.

Conforme mostrado na Tabela 1, os pacientes dos Grupos A e B possuíam mediana de idade semelhante, mas tinham proporções de gênero significativamente diferentes. Em relação aos fatores de risco cardiovascular e condições de comorbidade, não foram encontradas diferenças significativas na prevalência de diabetes mellitus e hipertensão, mas hiperlipidemia e doença 


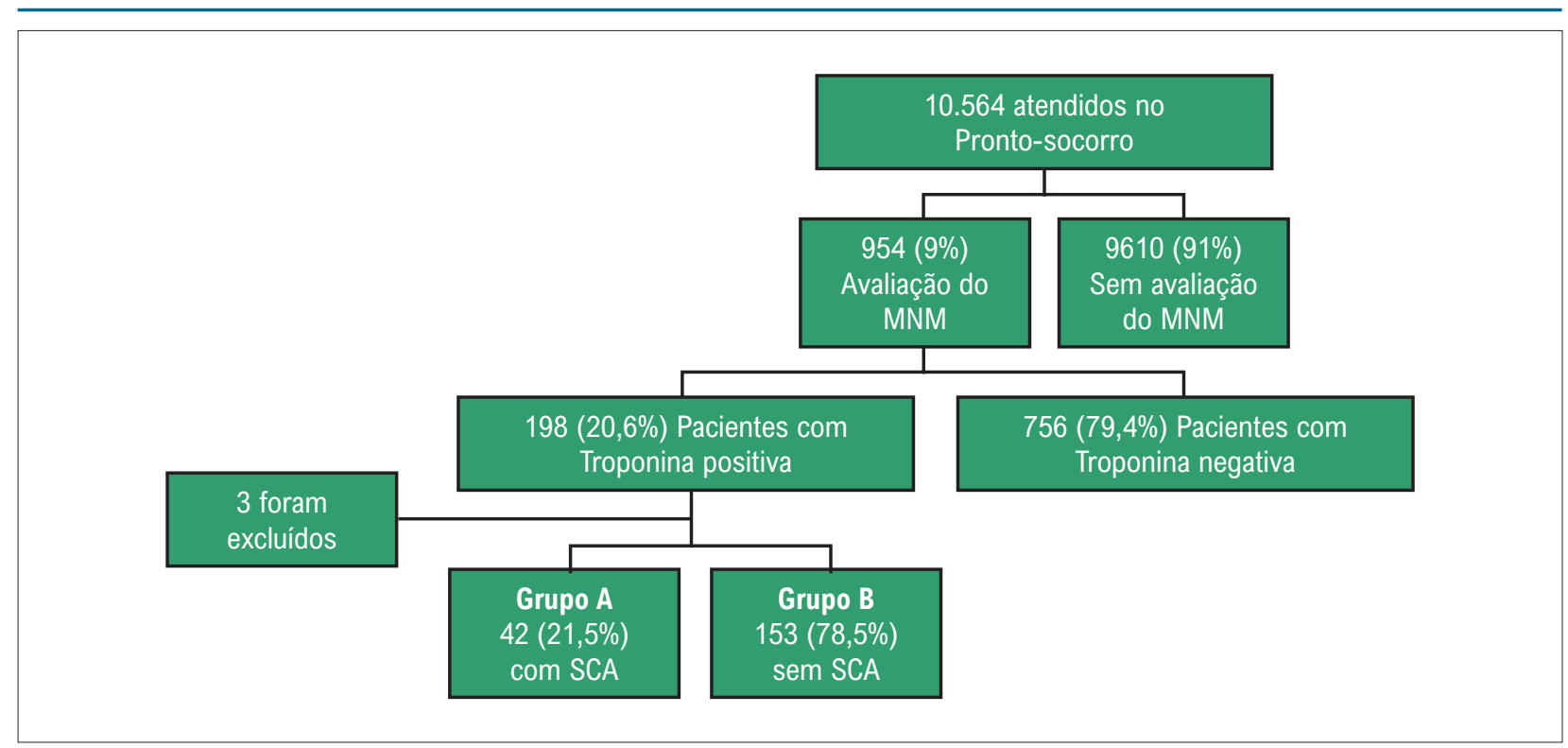

Figura 1 - Ilustração esquemática dos pacientes incluídos. MNM, marcadores de necrose miocárdica; SCA, síndrome coronariana aguda

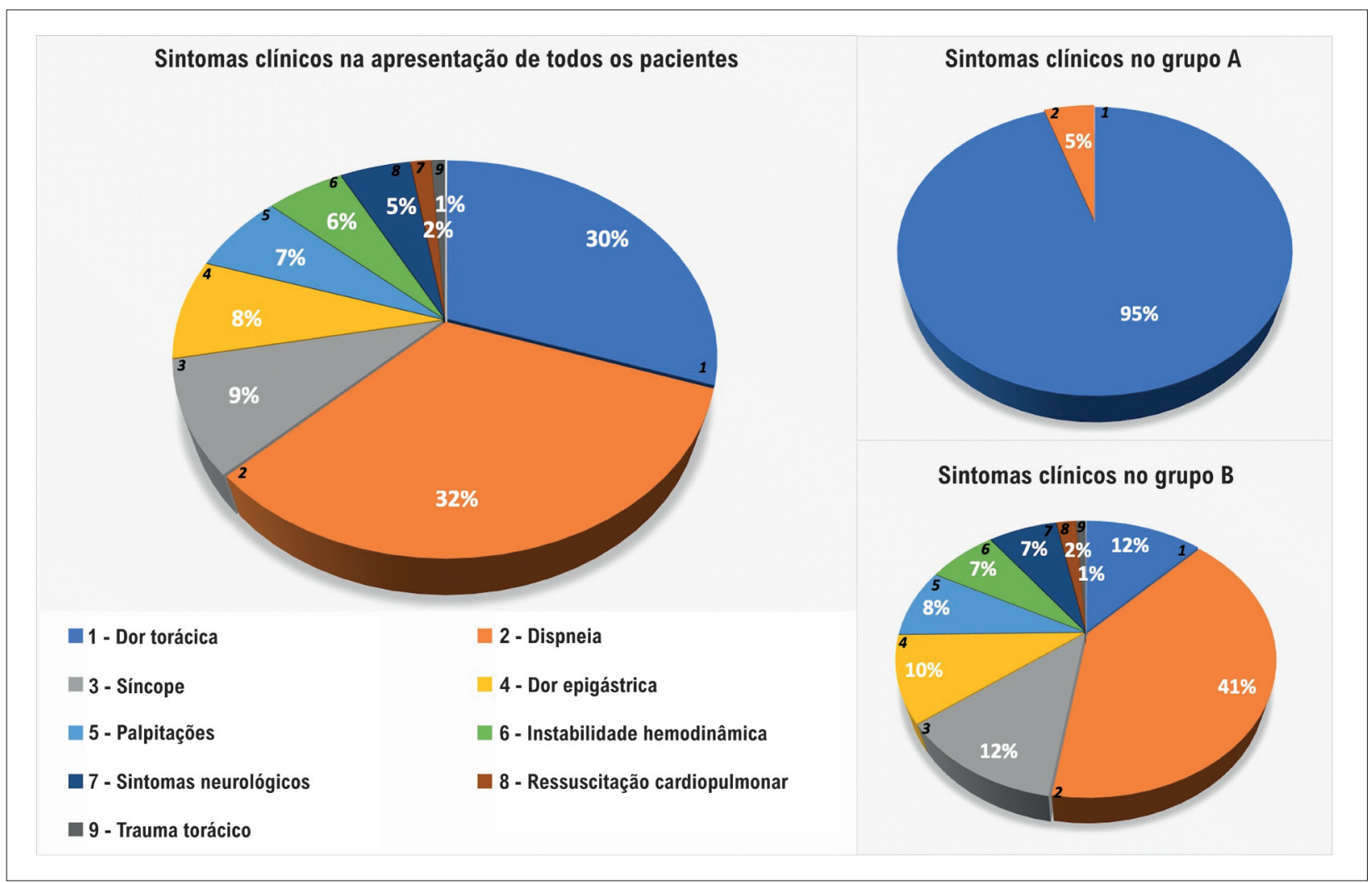

Figura 2 - Ilustração esquemática dos sintomas clínicos na apresentação de todos os pacientes e diferentes grupos A: com SCA e grupo B: Sem SCA.

arterial coronariana anterior foram mais comuns em pacientes com SCA e insuficiência cardíaca anterior e tratamento anticoagulante foram mais prevalentes em pacientes sem SCA.

Os principais diagnósticos dos pacientes do Grupo B foram miocardite /cardiomiopatia (40 [26\%]), seguido de infecção (celulite, pneumonia e pielonefrite, 24 [15,5\%]), arritmias agudas $(25[16,6 \%])$, doença renal crônica ou aguda (17 [11\%]), doença cerebral (13 [8,4\%]), sangramento abdominal ou gastrointestinal agudo (11[7,1\%]), embolia pulmonar $(6[3,9 \%])$, síncope inexplicada $(4[2,6 \%]])$, asma ou exacerbação de doença pulmonar obstrutiva crônica (4 $[2,6 \%])$ e outros (9 [6,5\%]). 


\begin{tabular}{|c|c|c|c|c|}
\hline & $\begin{array}{l}\text { Global } \\
\mathrm{N}=195\end{array}$ & $\begin{array}{c}\text { Grupo A } \\
n=42(22 \%)\end{array}$ & $\begin{array}{c}\text { Grupo B } \\
n=153(78 \%)\end{array}$ & p-valor \\
\hline Idade (anos), mediana (IIQ) & $77(21)$ anos & $71(19)$ anos & $78(19)$ anos & 0,06 \\
\hline Sexo masculino $\mathrm{n}(\%)$ & $105(53,8 \%)$ & $32(76,2 \%)$ & $73(47,7 \%)$ & 0,001 \\
\hline Diabetes mellitus, $n(\%)$ & $69(35,5 \%)$ & $16(37,2 \%)$ & $54(35 \%)$ & 0,89 \\
\hline Hipertensão, n (\%) & $155(79,3 \%)$ & $35(84,4 \%)$ & $119(78,1 \%)$ & 0,57 \\
\hline Hiperlipidemia, n (\%) & $87(44,4 \%)$ & $25(60 \%)$ & $61(40,1 \%)$ & 0,03 \\
\hline DAC anterior, $\mathrm{n}(\%)$ & $37(19 \%)$ & $13(31 \%)$ & $24(16 \%)$ & 0,02 \\
\hline IC anterior, $\mathrm{n}(\%)$ & $43(21,9 \%)$ & $9(21,9 \%)$ & $71(46,7 \%)$ & 0,02 \\
\hline $\mathrm{AVCl}$ anterior, $\mathrm{n}(\%)$ & $31(14,8 \%)$ & $3(6,3 \%)$ & $26(16,8 \%)$ & 0,26 \\
\hline TFG (ml/[min.1.73 m²]), mediana (IIQ) & $54(46)$ & $68(47)$ & $49(47)$ & 0,10 \\
\hline \multicolumn{5}{|l|}{ Medicação Anterior } \\
\hline Anticoagulantes & $62(33,7 \%)$ & $4(10 \%)$ & $47(31 \%)$ & 0,007 \\
\hline Antiplaquetários & $50(26,7 \%)$ & $15(37 \%)$ & $48(32 \%)$ & 0,13 \\
\hline Betabloqueadores & $69(36,9 \%)$ & $16(40 \%)$ & $53(36,1 \%)$ & 0,65 \\
\hline Inibidores ECA/ARA & $108(57,8 \%)$ & $23(57,5 \%)$ & $87(57,8 \%)$ & 0,97 \\
\hline ARM & $15(17,6 \%)$ & $2(12,5 \%)$ & $13(18,8 \%)$ & 0,55 \\
\hline Estatinas & $83(44,4 \%)$ & $24(60 \%)$ & $60(40,1 \%)$ & 0,02 \\
\hline Troponina padronizada na primeira avaliação *, n (\%) & & & & $<0,001$ \\
\hline $1-2,99$ & $95(48,9 \%)$ & $10(23,1 \%)$ & $85(55,8 \%)$ & \\
\hline $3-4,99$ & $36(18,3 \%)$ & $5(12,8 \%)$ & $31(19,7 \%)$ & \\
\hline $5-9,99$ & $19(9,7 \%)$ & $4(10,3 \%)$ & $15(9,7 \%)$ & \\
\hline $10+$ & $45(23,1 \%)$ & $23(53,8 \%)$ & $22(15 \%)$ & \\
\hline CK-MB elevada, $\mathrm{n}(\%)$ & $54(53 \%)$ & $22(53 \%)$ & $32(21 \%)$ & $<0,001$ \\
\hline Mioglobina elevada, n (\%) & $88(48 \%)$ & $23(56 \%)$ & $66(45 \%)$ & 0,24 \\
\hline CK-MB + Mioglobina elevadas, $\mathrm{n}(\%)$ & $43(22 \%)$ & $16(40 \%)$ & $25(16 \%)$ & 0,003 \\
\hline \% de elevação da troponina entre 2 medidas, mediana (IIQ) & $7(73)$ & $183(666)$ & $2,65(42)$ & $<0,001$ \\
\hline
\end{tabular}

\section{Dados de desfechos}

A mediana do seguimento (IIQ) foi de 477 dias (316). Não foram encontradas diferenças significativas entre os grupos em relação à mortalidade intra-hospitalar (6 [14,3\%] vs. 21 [13,7\%], $p=0,077)$, mortalidade em 30 dias $(6[14,3 \%]$ vs. $27[17,6 \%], p=0,4)$ e reinternação cardiovascular no seguimento (11 [29,7\%] vs. 32 [24,2\%], $\mathrm{p}=0,316)$. Notavelmente, a taxa de mortalidade em longo prazo foi significativamente maior nos pacientes do grupo B (9 [21,4\%] vs. 59 [38,6\%], p = 0,039), embora as curvas de sobrevivência dos dois grupos não fossem significativamente diferentes (log rank, 3,45; p = $0,063)$. As principais causas de morte do grupo B foram: cardiovasculares em 12 indivíduos (nenhum deles com diagnóstico de infarto agudo do miocárdio), 27 nãocardiovasculares, 13 por causas desconhecidas e 4 mistas de causas cardiovasculares e outras comorbidades.

\section{Resultados principais}

Os preditores de mortalidade dos pacientes do Grupo B estão descritos na Tabela 2. Foi observado que maior idade ( $p$ $<0,001)$, insuficiência cardíaca anterior $(p=0,049)$, medicação antiplaquetária anterior ( $p=0,005)$ e hospitalização após a avaliação índice do PS ( $p<0,001)$ foram preditores de mortalidade. A morte no seguimento não foi relacionada com os níveis de Tncl, CK-MB ou mioglobina (primeira, segunda ou ambas as avaliações). No entanto, a elevação isolada da troponina (isto é, sem elevação concomitante da CK-MB ou mioglobina) foi um poderoso preditor de sobrevivência; de fato, a elevação isolada da troponina na primeira medida estava presente em $58,7 \%$ dos sobreviventes vs. $40 \%$ dos não sobreviventes ( $p=0,021)$; os pacientes com duas medidas isoladas de elevação da troponina tinham maior probabilidade de sobreviver (48 [53,9\%] dos sobreviventes vs. 8 [17,4\%] dos não sobreviventes [p $<0,001])$. 


\begin{tabular}{|c|c|c|c|}
\hline & $\begin{array}{l}\text { Sobreviventes } \\
\quad(n=94)\end{array}$ & $\begin{array}{l}\text { Não-sobreviventes ( } \mathrm{n} \\
=59 \text { ) }\end{array}$ & p-valor \\
\hline Idade, mediana (IIQ) & $76(24)$ anos & $84(13)$ & $<0,001$ \\
\hline Sexo masculino, $n(\%)$ & $44(46,8 \%)$ & $29(49,2 \%)$ & 0,77 \\
\hline \multicolumn{4}{|l|}{ Fatores de risco CV, $\mathrm{n}(\%)$} \\
\hline Diabetes mellitus & $28(30,8 \%)$ & $22(38,6 \%)$ & 0,33 \\
\hline Hipertensão & $68(73,9 \%)$ & $44(78,6 \%)$ & 0,52 \\
\hline DAC anterior & $14(15,2 \%)$ & $9(16,4 \%)$ & 0,85 \\
\hline HF anterior & $33(39,3 \%)$ & $31(56,4 \%)$ & 0,049 \\
\hline TFG, mL / (min.1.73 m²), mediana (IIQ) & $56(48)$ & $45(34)$ & 0,05 \\
\hline Frequência cardiaca, bpm, mediana (IIQ) & $75(33)$ & $84(36)$ & 0,10 \\
\hline \multicolumn{4}{|l|}{ Medicação Anterior, n (\%) } \\
\hline Antiplaquetária & $22(23,9 \%)$ & $25(35,5 \%)$ & 0,02 \\
\hline Anticoagulantes & $34(37 \%)$ & $12(21,8 \%)$ & 0,06 \\
\hline Betabloqueadores & $35(38 \%)$ & $18(32,7 \%)$ & 0,52 \\
\hline Inibidor de ECA & $57(62 \%)$ & $28(50,9 \%)$ & 0,19 \\
\hline ARM & $9(20,5 \%)$ & $4(16 \%)$ & 0,65 \\
\hline Estatinas & $36(39,1 \%)$ & $23(41,8 \%)$ & 0,75 \\
\hline $\begin{array}{l}\text { ECG, } \mathbf{n}(\%) \\
\text { Sem alterações significativas }\end{array}$ & $51(64,6 \%)$ & $16(50 \%)$ & 0,38 \\
\hline Elevação do ST & $1(1,3 \%)$ & $0(0 \%)$ & \\
\hline Depressão do ST ou onda T negativa & $16(20,3 \%)$ & $6(18,8 \%)$ & \\
\hline Fibrilação atrial & $28(35,0 \%)$ & $18(46,2 \%)$ & \\
\hline BRE & $4(5,1 \%)$ & $4(12,5 \%)$ & \\
\hline Ritmo & $4(5,1 \%)$ & $3(9,4 \%)$ & \\
\hline \multicolumn{4}{|l|}{ Marcadores de necrose miocárdica, mediana (IIQ) } \\
\hline Troponina $(\mathrm{ng} / \mathrm{mL})$ na primeira avaliação & $0,13(0,23)$ & $0,10(0,18)$ & 0,61 \\
\hline CK-MB $(\mathrm{ng} / \mathrm{mL})$ na primeira avaliação & $1,6(1,8)$ & $1,9(2,05)$ & 0,50 \\
\hline Mioglobina ( $\mathrm{ng} / \mathrm{mL}$ ) na primeira avaliação & $70(120)$ & $99(175)$ & 0,06 \\
\hline Elevação isolada da troponina na primeira avaliação, $n$ (\%) & $54(58,7 \%)$ & $22(40 \%)$ & 0,028 \\
\hline Troponina (ng / mL) na segunda avaliação, mediana (IIQ) & $0,12(0,16)$ & $0,14(0,32)$ & 0,28 \\
\hline $\begin{array}{l}\% \text { de variação da troponina I em duas medidas } \\
\text { sequenciais, mediana (IIQ) }\end{array}$ & $0(32)$ & $27(35)$ & 0,002 \\
\hline $\begin{array}{l}\text { Elevação isolada da troponina em duas medidas } \\
\text { sequenciais, } \mathrm{n}(\%)\end{array}$ & $48(53,9 \%)$ & $8(17,4 \%)$ & $<0,001$ \\
\hline Hospitalização no evento índice, n (\%) & $52(55,3 \%)$ & $51(86,4 \%)$ & $<0,001$ \\
\hline Revascularização coronária & & & 0,88 \\
\hline Sem terapia específica, n (\%) & $88(93,6 \%)$ & $54(91,5 \%)$ & \\
\hline TMO, n (\%) & $5(5,3 \%)$ & $4(6,8 \%)$ & \\
\hline ICP + TMO, n (\%) & $1(1,1 \%)$ & $1(1,1 \%)$ & \\
\hline
\end{tabular}

IIQ: intervalo interquartil; DAC: doença arterial coronariana; IC: insuficiência cardiaca; AVCl: acidente vascular cerebral isquêmico agudo; TFG: taxa de filtração glomerular de acordo com a equação MDRD; Inibidor da ECA: inibidor da enzima conversora da angiotensina; ARM: antagonista do receptor mineralocorticoide; ECG: eletrocardiograma; CK: creatina-quinase; CK-MB: creatina quinase-MB; TMO: terapia médica otimizada; ICP: intervenção coronária percutânea; 
A análise de regressão de Cox é apresentada na Tabela 3. A análise univariada mostrou que em pacientes internados com troponina isolada em duas medidas consecutivas de MNM, a probabilidade de sobrevida em longo prazo no seguimento aumentou em quatro vezes $(p<0,001)$. A análise multivariada de Cox corrigida para idade e sexo demonstrou que a elevação isolada da troponina em duas medidas consecutivas permaneceu um preditor independente de sobrevida (HR, 0,433; IC95\%, 0,196-0,958; $p=0,039$ ). A Figura 3 mostra as curvas de sobrevida dos pacientes do Grupo B de acordo com a presença de elevação isolada da troponina em duas medidas consecutivas (log rank, 18,09; $\mathrm{p}<0,001)$.

\section{Discussão}

\section{Interpretação}

Em nosso estudo, 78\% dos pacientes com elevação da troponina receberam diagnóstico de não-SCA, o que está de acordo com estudos anteriores. ${ }^{20,21} \mathrm{Em}$ algumas séries, a isquemia miocárdica não foi identificada em aproximadamente $65 \%$ dos pacientes do PS com elevação da troponina. O espectro do diagnóstico clínico foi extremamente heterogêneo em nosso estudo, englobando condições de alto risco. O prognóstico a médio prazo foi claramente pior para pacientes com troponina elevada e sem SCA do que para pacientes com níveis normais de troponina. Provavelmente também foi pior do que para pacientes com diagnóstico de SCA, com esses achados relatados em estudos anteriores. ${ }^{16,22,23}$

Neste estudo, 33,5\% dos pacientes com níveis elevados de troponina e diagnóstico de não-SCA tiveram alta do PS sem internação. Esse percentual parece muito alto, mas taxas mais elevadas foram descritas por outros autores. ${ }^{21,24,25} \mathrm{No}$ grupo sem SCA, a mortalidade em 16 meses foi de 38,6\%, mas $81,4 \%$ dessas mortes ocorreram durante a internação ou nos primeiros 30 dias, o que reforça o papel do MNM como preditor de mortalidade nesse grupo. Considerando a alta taxa de alta hospitalar após uma internação no PS e o alto risco de mortalidade conferido pela elevação da troponina, não é surpreendente que os pacientes idosos com elevação da troponina que recebem um diagnóstico diferente de SCA e não são hospitalizados apresentem em um risco inaceitável de morte.

Acreditamos que a alta taxa de mortalidade durante a hospitalização e seguimento está intimamente relacionada à idade avançada e maiores comorbidades (insuficiência cardíaca anterior ou medicação antiplaquetária), conforme relatado por outros autores. ${ }^{21}$

O único biomarcador recomendado para uso no diagnóstico de SCA neste momento é a Tnc, devido à sua sensibilidade e precisão superiores. ${ }^{2,3}$ De fato, até $80 \%$ dos pacientes com IM isquêmico terá um nível elevado de troponina nas 2 a 3 horas após a chegada ao PS. ${ }^{7}$

Nosso estudo é notável por descobrir que a elevação isolada da Tncl em duas análises consecutivas de MNMs é um preditor de sobrevida para pacientes com elevação da Tncl e sem SCA, em comparação com a elevação de pelo menos dois MNMs (Tncl e CK-MB e/ou mioglobina). Algumas particularidades das diferentes propriedades das moléculas de MNM poderiam explicar esse fato. A mioglobina apresenta liberação precoce e clearance rápida (liberada a partir de $1 \mathrm{~h}$ após a lesão e retorna à linha de base em 24-36h), enquanto a CK-MB apresenta uma liberação e clearance mais lentas (liberada a partir de 4-9h após a lesão e clearance em $48-72 \mathrm{~h}),{ }^{26}$ e a troponina apresenta liberação semelhante à da CK-MB (4-9h), mas clearance retardada (7-10 dias). ${ }^{27}$ Nossa hipótese é que a elevação persistente da CK-MB e/ou mioglobina junto com a troponina em duas análises consecutivas de MNM implica em uma lesão miocárdica recente ou permanente, mesmo em pacientes sem SCA.

Provavelmente houve uma diferença no mecanismo de liberação de diferentes moléculas de MNM de acordo com o tipo e a gravidade da lesão. Alguns estudos em animais e células humanas sugeriram que a descarga de proteínas miocárdicas, exatamente como a Tnc, pode não implicar em necrose miocárdica. ${ }^{28}$

Em relação às subunidades $\mathrm{T}$ e I, a Tncl tem um peso molecular de $37 \mathrm{kDa}$ e a TncT tem um peso molecular de 21 $\mathrm{kDa}$, ambas presentes principalmente nos sarcômeros e 4-6\% no citoplasma. Após a lesão miocárdica, a troponina citosólica é liberada primeiro; à medida que mais danos ocorrem, a troponina presente no sarcômero é liberada na circulação; ${ }^{8}$ estudos anteriores defenderam a ideia de que cardiomiócitos com lesão reversível poderiam liberar troponina. ${ }^{29-31}$

A CK-MB também é liberada com necrose tecidual devido ao seu alto peso molecular (86kDa). A mioglobina tem liberação rápida, provavelmente pelo baixo peso molecular (17kDa) e sua localização citoplasmática, podendo ser liberada sob estresse miocárdico sem necrose, ${ }^{8}$ assim como a troponina.

Essa propriedade molecular de diferentes MNMs poderia explicar a incapacidade de uma medida isolada da troponina I em predizer mortalidade no presente estudo e em outro. ${ }^{32}$ Este achado destaca o papel valioso da CK-MB e da mioglobina que não pode ser realizado pela medida isolada da troponina. No entanto, as diretrizes atuais recomendam que a Tnc seja o único biomarcador utilizado para o diagnóstico da SCA, devido à sua sensibilidade e precisão superiores. ${ }^{2,3}$ No entanto, a não-realização das medidas de CK-MB e mioglobina pode ter um custo, especialmente para aqueles pacientes com um diagnóstico de não-SCA. Acreditamos que a exclusão da CK-MB e mioglobina da avaliação de rotina com MNMs em muitas instituições e diretrizes deve ser reconsiderada, devido ao seu valor prognóstico adjuvante superior, principalmente em pacientes sem SCA, e o aumento do número de pacientes com elevação da troponina que serão observados com níveis de troponinas altamente sensíveis.

\section{Limitações}

Nossa instituição segue um protocolo não-restritivo para pedidos de medidas de MNM no PS. Portanto, nossa taxa de pacientes sem SCA estava provavelmente aumentado em comparação com protocolos mais rígidos.

Nosso estudo analisou a mortalidade dos pacientes sem considerar que os pacientes foram tratados de forma diferente de acordo com o diagnóstico inicial. Esta pode parecer uma 
Tabela 3 - Análise de regressão de Cox univariada e multivariada (corrigida para idade e sexo) das variáveis clínicas e sobrevida em longo prazo de pacientes com elevação da troponina e síndrome coronariana não-aguda

\begin{tabular}{|c|c|c|c|c|}
\hline & \multicolumn{2}{|c|}{ Regressão univariada de Cox } & \multicolumn{2}{|c|}{ Regressão multivariada de Cox } \\
\hline & HR (IC95\%) & p-valor & HR (IC95\%) & p-valor \\
\hline Idade, anos & $1,040(1,017-1,063)$ & 0,001 & $1,030(1,002-1,058)$ & 0,038 \\
\hline Sexo & $0,899(0,540-1,499)$ & 0,684 & $0,807(0,436-1,493)$ & 0,494 \\
\hline \multicolumn{5}{|l|}{ Fatores de risco CV } \\
\hline Diabetes mellitus & $1,336(0,783-2,277)$ & 0,288 & & \\
\hline Hipertensão & $1,245(0,658-2,358)$ & 0,501 & & \\
\hline DAC anterior & $1,067(0,521-2,182)$ & 0,860 & & \\
\hline $\mathrm{HF}$ anterior & $1,649(0,967-2,812)$ & 0,066 & & \\
\hline GFR, mL / (min.1.73 m²) & $0,992(0,984-1,001)$ & 0,082 & & \\
\hline Frequência cardíaca, bpm & $1,006(0,997-1,014)$ & 0,193 & & \\
\hline \multicolumn{5}{|l|}{ Medicação Anterior } \\
\hline Antiplaquetários & $1,867(1,230-2,835)$ & 0,006 & $1,823(1,105-3,006)$ & 0,019 \\
\hline Betabloqueadores & $0,806(0,459-1,416)$ & 0,449 & & \\
\hline Inibidor de ECA & $0,689(0,406-1,170)$ & 0,170 & & \\
\hline ARM & $0,764(0,262-2,226)$ & 0,611 & & \\
\hline Estatinas & $1,017(0,595-1,739)$ & 0,950 & & \\
\hline Padrão de ECG & $1,162(0,999-1,351)$ & 0,067 & & \\
\hline \multicolumn{5}{|l|}{ Laboratório } \\
\hline Elevação isolada da troponina na primeira avaliação & $0,533(0,311-0,916)$ & 0,021 & $1,097(0,378-3,180)$ & 0,865 \\
\hline Elevação isolada da troponina na segunda avaliação & $0,528(0,218-1,279)$ & 0,142 & & \\
\hline \% de elevação da troponina em duas avaliações sequenciais & $1,000(0,999-1,001)$ & 0,750 & & \\
\hline Elevação isolada da troponina em duas avaliações sequenciais & $0,239(0,111-0,512)$ & $<0,001$ & $0,433(0,196-0,958)$ & 0,039 \\
\hline Hospitalização no evento índice & $3.782(1.794-7.973)$ & $<0.001$ & $4.708(1.652-13.423)$ & 0.004 \\
\hline
\end{tabular}

IC 95\%: intervalo de confiança de 95\%; DAC: doença arterial coronariana; IC: insuficiência cardiaca; TFG: taxa de filtração glomerular de acordo com a equação MDRD; CK: creatina quinase; CK-MB: creatina quinase-MB; Inibidor da ECA: inibidor da enzima conversora da angiotensina; ARM: antagonista do receptor mineralocorticoide; ECG: eletrocardiograma.

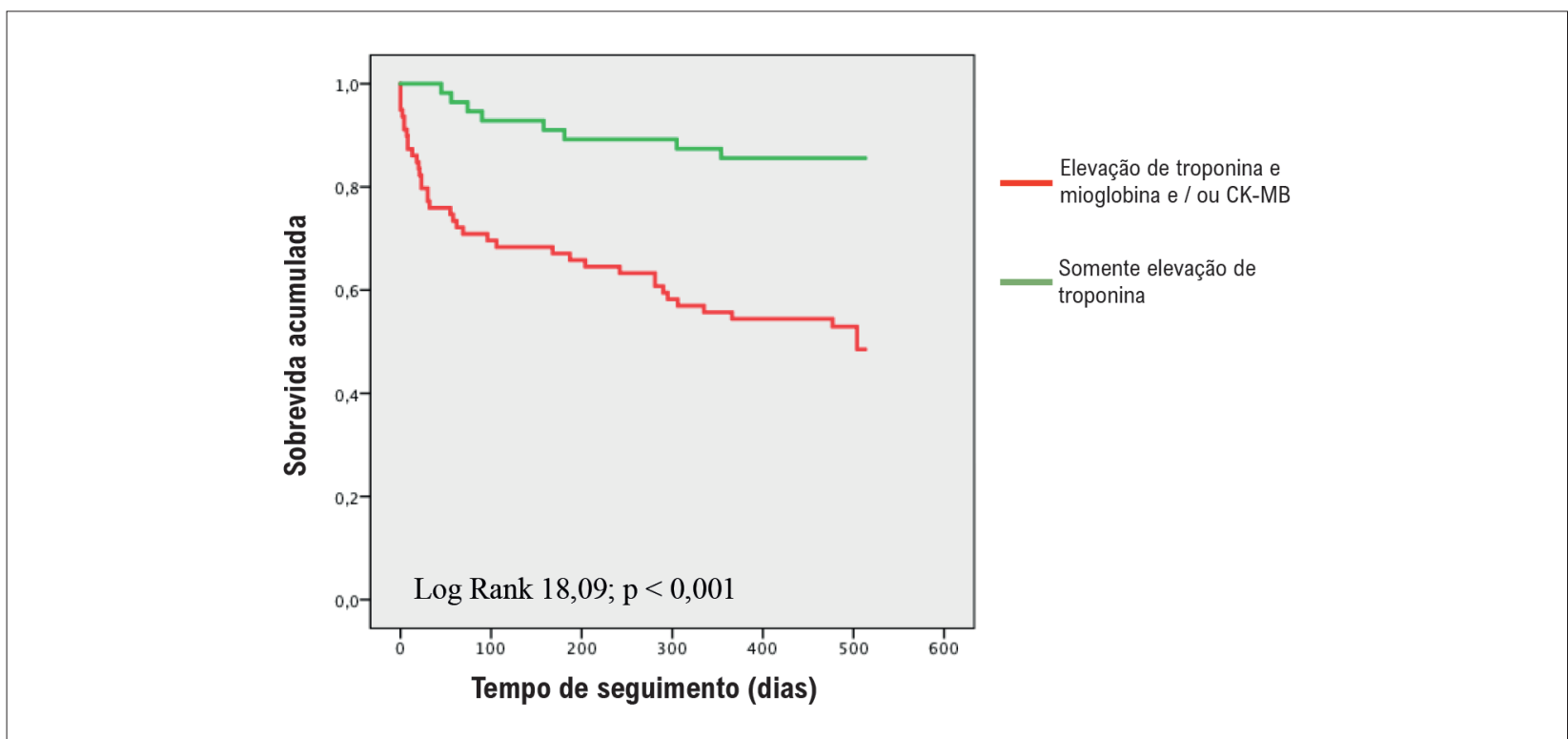

Figura 3 - Curva de sobrevida de Kaplan-Meier no seguimento de 16 meses de pacientes não-SCA de acordo com resultados de duas avaliações consecutivas de MNM. 
limitação importante, mas deve ser esclarecido que cada processo clínico geralmente tem seu próprio manejo específico que influencia o prognóstico do paciente. Portanto, o prognóstico dos grupos é inerente, de alguma forma, ao manejo fornecido. Por exemplo, os pacientes com SCA são geralmente admitidos para tratamento com agentes antiplaquetários, anticoagulantes, estatinas, revascularização e outras terapias, e essa abordagem tem um prognóstico específico.

Os dados foram coletados retrospectivamente, sendo possível que alguns prontuários estivessem incompletos e o histórico clínico subvalorizado.

O ensaio de troponina utilizado anteriormente em nosso hospital era um ensaio contemporâneo denominado 'troponina sensível' e não era altamente sensível, ao contrário do ensaio de troponina utilizado atualmente, através do qual espera-se detectar valores positivos de troponina em mais pacientes, como descrito para este ensaio de troponina. ${ }^{18,19}$

\section{Conclusão}

Uma alta porcentagem de pacientes com um nível elevado de troponina medido no PS não foi diagnosticada com SCA. Esses pacientes apresentavam perfil clínico de alto risco, ampla heterogeneidade em relação ao diagnóstico principal e prognóstico adverso aos 16 meses. Uma elevação isolada da troponina I em duas determinações consecutivas de MNMs foi um forte preditor de sobrevida em pacientes sem SCA com elevação da troponina.

\section{Agradecimentos}

Gostaríamos de agradecer a todos os médicos e enfermeiras envolvidos no tratamento desses pacientes e seus registros.

\section{Referências}

1. Thygesen K, Alpert JS, Jaffe AS, Chaitman BR, Bax JJ, Morrow DA, et al. Fourth universal definition of myocardial infarction (2018). Circulation. 2018;138(20):e618-51.

2. Amsterdam EA, Wenger NK, Brindis RG, Casey DE, Ganiats TG, Holmes DR, et al. 2014 AHA/ACC guideline for the management of patients with non-stelevation acute coronary syndromes: a report of the American College of Cardiology/American Heart Association Task Force on Practice Guidelines. Circulation. 2014;130(25):2354-94.

3. Roffi M, Patrono C, Collet JP, Mueller C, Valgimigli M, Andreotti F, et al. 2015 ESC guidelines for the management of acute coronary syndromes in patients presenting without persistent st-segment elevation: task force for the management of acute coronary syndromes in patients presenting without persistent ST-segment elevation of the European Society of Cardiology (ESC). Eur HeartJ. 2016;37(3):267-315

4. Lewandrowski K, Chen A, Januzzi J. Cardiac markers for myocardial infarction. A brief review. Am J Clin Pathol. 2002;118(Suppl):93-9.

5. Katus HA, Remppis A, Scheffold T, Diederich KW, Kuebler W. Intracellular compartmentation of cardiac troponin $\mathrm{T}$ and its release kinetics in patients with reperfused and nonreperfused myocardial infarction. Am J Cardiol. 1991;67(16):1360-7.

6. Higgins JP, Higgins JA. Elevation of cardiac troponin I indicates more than myocardial ischemia. Clin Invest Med. 2003;26(3):133-47.
Também agradecemos o Departamento do Laboratório por fornecer a lista de pacientes com elevação da troponina, a base de dados inicial dos pacientes.

\section{Contribuição dos autores}

Concepção e desenho da pesquisa: Domingues C, Ferreira MJV, Ferreira JM; Obtenção de dados: Domingues C, Marinho AV, Alves PM, Ferreira C; Análise e interpretação dos dados: Domingues C, Ferreira MJV, Marinho AV, Alves PM, Fonseca I, Gonçalves L; Análise estatística: Domingues C, Ferreira MJV, Ferreira JM, Ferreira C; Redação do manuscrito: Domingues C, Ferreira MJV; Revisão crítica do manuscrito quanto ao conteúdo intelectual importante: Ferreira MJV, Ferreira JM, Marinho AV, Alves PM, Ferreira C, Fonseca I, Gonçalves L.

\section{Potencial conflito de interesses}

Declaro não haver conflito de interesses pertinentes.

\section{Fontes de financiamento}

O presente estudo não teve fontes de financiamento externas.

\section{Vinculação acadêmica}

Não há vinculação deste estudo a programas de pósgraduação.

\section{Aprovação ética e consentimento informado}

Este artigo não contém estudos com humanos ou animais realizados por nenhum dos autores.

7. White HD. Pathobiology of troponin elevations: do elevations occur with myocardial ischemia as well as necrosis? J Am Coll Cardiol. 2011;57(24):2406-8.

8. Santaló M, Martin A, Velilla J, Povar J, Temboury F, Balaguer J, et al. Using high-sensitivity troponin T: the importance of the proper gold standard. Am J Med. 2013;126(8):709-17.

9. Kehl DW, Iqbal N, Fard A, Kipper BA, Landa AP, Maisel AS. Biomarkers in acute myocardial injury. Transl Res. 2012;159(4):252-64.

10. Sheyin O, Davies O, Duan W, Perez X. The prognostic significance of troponin elevation in patients with sepsis: a meta-analysis. Heart Lung. 2015;44(1):75-81.

11. Michos ED, Wilson LM, Yeh HC, Berger Z, Suarez-Cuervo C, Stacy SR, et al Prognostic value of cardiac troponin in patients with chronic kidney disease without suspected acute coronary syndrome: a systematic review and metaanalysis. Ann Intern Med. 2014;161(7):491-501.

12. Sarkisian L, Saaby L, Poulsen TS, Gerke O, Jangaard N, Hosbond S, et al. Clinical characteristics and outcomes of patients with myocardial infarction, myocardial injury, and nonelevated troponins. Am J Med. 2016;129(4):446e.5-e21.

13. Shah KS, Maisel AS, Fonarow GC. Troponin in heart failure. Heart Fail Clin 2018;14(1):57-64. 


\section{Artigo Original}

14. Wu C, Singh A, Collins B, Fatima A, Qamar A, Gupta A, et al. Causes of troponin elevation and associated mortality in young patients. Am J Med. 2018;131(3):284-92.

15. Wong P, Murray S, Ramsewak A, Robinson A, Heyningen C, Rodrigues E. Raised cardiac troponin T levels in patients without acute coronary syndrome. Postgrad Med J. 2007;83(977):200-5.

16. Bardají A, Cediel G, Carrasquer A, Castro R, Sánchez R, Boqué C. Troponin elevation in patients without acute coronary syndrome. Rev Esp Cardiol. 2015;68(6):469-76.

17. Calderón JLM, Pérez JMV, Bustos F, Gómez JCS. Performance characteristics of loci method for measuring cardiac troponin I on the dimension EXL. Pract Lab Med. 2015 Apr 8;1:42-7.

18. Javed U, Aftab W, Ambrose JA, Wessel RJ, Mouanoutoua M, Huang G, et al. Frequency of elevated troponin $i$ and diagnosis of acute myocardial infarction. Am J Cardiol. 2009;104(1):9-13.

19. Brunner NW, Scheuermeyer FX, Grafstein E, Ramanathan K. Outcomes of non-acute coronary syndrome patients discharged from the emergency department with troponin positivity. Can J Emerg Med. 2014;16(1):41-52.

20. Schreiber D, Miller SM. Cardiac markers: definition and efficacy. Medscape [Internet]; 2018. [acesso 29 jan 2021]. Disponível em: https:// emedicine.medscape.com/article/811905-over.

21. Vaidya HC. Myoglobin: an early biochemical marker for the diagnosis of acute myocardial infarction. J Clin Immunoass. 1994;17:35-9.

22. Tucker JF, Collins RA, Anderson AJ, Hauser J, Kalas J AF. Early diagnostic efficiency of cardiac troponin I and troponin T for acute myocardial infarction. Acad Emerg Med. 1997; 4(1):13-21.
23. Mair J, Lindahl B, Hammarsten O, Müller C, Giannitsis E, Huber K, et al. How is cardiactroponin released from injured myocardium? Eur HeartJ AcuteCardiovasc Care. 2018;7(6):553-60.

24. Vaidya HC, Vaananen HK. Myoglobin and carbonic anhydrase III. In: Cardiac markers. Totowa: Humana Press; 2011. p. 103-12. (Pathology and Laboratory Medicine book series).

25. Wahed A, Dasgupta A. Clinical chemistry, immunology and laboratory quality control. Amsterdam: Elsevier; 2014. Chapter 8, Cardiac markers; p. 127-41.

26. Mair. Tissue release of cardiac markers: from physiology to clinical applications. Clin Chem Lab Med. 1999;37(11-12):1077-84

27. Piper HM, SchwartzP,Spahr R, HütterJF, Spieckermann PG. Earlyenzyme release from myocardial cells is not due to irreversible cell damage. J Mol Cell Cardiol. 1984;16(4):385-8.

28. Demonbreun AR, MCNally EM. Plasma membrane repair in health and disease. Curr Top Membr. 2016;77:67-96.

29. Kavasoglu M, Eken C, Eray O, Serinken M, Gulen B. Value of high-sensitive cardiac troponin in predicting mortality in the emergency department. Clin Lab. 2016;62(8):1483-9

30. JafferyZ, Nowak R, Khoury N, Tokarski G, Lanfear DE, Jacobsen G, etal. Myoglobin and troponin I elevation predict 5 -year mortality in patients with undifferentiated chest pain in the emergency department. Am HeartJ. 2008;156(5):939-45.

31. Croce A, Brunati P, Colzani C, Terramocci R, Favero S, Bordoni G, et al. A rational adoption of the high sensitive assay for cardiac troponin I in diagnostic routine. Dis Markers. 2017;2017:4523096.

32. Reichlin T, Twerenbold R, Reiter M, Steuer S, Bassetti S, Balmelli C, et al. Introduction of high-sensitivity troponin assays: impact on myocardial infarction incidence and prognosis. Am J Med. 2012;125(12):1205-13. 